\title{
Sustentabilidade ambiental da água consumida no Município do Rio de Janeiro, Brasil
}

\author{
Aldo Ferreira ${ }^{1}$ e Cynara Cunha ${ }^{1}$
}

Como citar Ferreira A, Cunha C. Sustentabilidade ambiental da água consumida no Município do Rio de Janeiro, Brasil. Rev Panam Salud Publica. 2005;18(1):93-99.

RESUMO Objetivo. Propor um índice de sustentabilidade da água de uso urbano com indicadores que possam fundamentar o desenvolvimento de ações planejadas.

Métodos. Foram selecionados 11 indicadores (incluindo aspectos relativos a água e esgoto) com base nos parâmetros do Sistema Nacional de Informação sobre Saneamento (SNIS) para os anos-base 2000 e 2001. Para cada indicador foi atribuída uma nota e arbitrado um peso. A partir disso, foi calculado o indice de sustentabilidade da água urbana (ISAU), utilizando-se a fórmula ISAU = produto $\left(I_{i}^{p i}\right)$, onde $I_{i}$ é a nota para cada indicador $i$, variando entre 0 e 100; e $p_{i}$ é o peso para cada indicador $i$, sendo $\sum_{i=1}^{n} p_{i}=1$, onde $n$ é o número de indicadores considerados. A pior qualidade da água é indicada por um valor igual a zero, e a melhor, por um valor igual a 100.

Resultados. O valor do índice de sustentabilidade da água urbana do Município do Rio de Janeiro foi de 58,99 e 59,57 para os anos de 2000 e 2001, respectivamente, valores que indicam qualidade boa da água.

Conclusões. A melhora observada na qualidade da água no Rio de Janeiro possivelmente se deve à implantação, na década de 1990, de um programa de recuperação ambiental na região. O planejamento ambiental para uso racional e prevenção da destruição dos recursos hídricos é fundamental para a manutenção da sociedade.

Palavras-chave Abastecimento de água, poluição da água, purificação da água, saúde ambiental, gerenciamento de resíduos, conservação de recursos naturais.

A água, um recurso indispensável para a sobrevivência de todas as espécies, exerce uma influência decisiva na

\footnotetext{
1 Fundação Instituto Oswaldo Cruz, Escola Nacional de Saúde Pública, Rio de Janeiro (RJ), Brasil. Enviar correspondência para Aldo Ferreira no seguinte endereço: Rua Leopoldo Bulhões 1480, Manguinhos, CEP 21041-210, Rio de Janeiro, RJ, Brasil. Fone: +55-21-2598-2746; e-mail: aldoferreira@ fiocruz.br
}

qualidade de vida das populações. Contudo, o modo como são utilizados e gerenciados os recursos hídricos tem levado a um nível de degradação ambiental e a um risco de escassez de água que comprometem a qualidade de vida das gerações futuras. Ao longo das décadas, a degradação ambiental se deu de diferentes formas. Na década de 1950, foi marcante a depleção de oxigênio; na década de 1960, a eu- troficação; nos anos 1970, a poluição por metais pesados; nos 1980, o uso excessivo de micropoluentes orgânicos e pesticidas; nos 1990, a contaminação da água subterrânea; e a década de 2000 está sendo marcada pela escassez da água (1).

A gestão da água e do meio associado, nomeadamente os solos, está fortemente ligada à evolução e à experiência do planejamento. Nas ultimas 
décadas, o paradigma ambiental ampliou significativamente o conceito de abastecimento de água integrado aos efeitos das intervenções e das ações de gestão nos ecossistemas associados e à defesa de aspectos especiais da natureza. Em outras palavras, cresceu a importância da prevenção contra a poluição, sendo, atualmente, a qualidade ecológica um dos parâmetros a se ter em conta no planejamento da gestão e uso da água. O entendimento do recurso natural água como um bem econômico e finito deve fazer com que todos os atores a utilizem de forma a maximizar o bem-estar social, seja produzindo com a máxima eficiência, seja consumindo sem desperdícios.

O planeta Terra é finito; e, nesse sentido, há limitações para o crescimento populacional, principalmente no ritmo atual, de mais de $1,5 \%$ ao ano (o que representa quase 100 milhões de pessoas todos os anos). Os dados disponíveis mostram que, até 2025, mais de 3 bilhões de pessoas se somarão aos atuais 7 bilhões, o que agravará os problemas ambientais no contexto das cidades e representará um grande desafio para os gestores urbanos (2). Agrega-se a isso o fato de que 1,7 bilhão de pessoas, ou um terço da população do mundo em desenvolvimento, vivem em países que enfrentam tensão quanto ao suprimento de água (ou seja, consomem mais de $20 \%$ da sua oferta de água renovável a cada ano). Se as tendências atuais persistirem, esse número pode chegar a 5 bilhões até 2025. O acesso limitado à água enfraquece as perspectivas de desenvolvimento de muitos países, e os conflitos causados pela utilização e distribuição da água são uma causa vulgar de disputas internacionais (1).

As grandes concentrações urbanas brasileiras apresentam condições críticas de sustentabilidade, devido ao excesso de cargas de poluição doméstica e industrial e à ocorrência de enchentes urbanas, que contaminam os mananciais, além de uma forte demanda de água (3). Visualiza-se, assim, uma iminente redução na disponibilidade hídrica. Esse processo ocorre, entre outros fatores, pela dificuldade que os municípios enfrentam no gerenciamento e na percepção integrada do ambiente. Repercute, conseqüentemente, na população, uma degradação da qualidade de vida, com o agravamento de doenças emergentes e reemergentes de veiculação hídrica e o colapso das atividades comerciais e industriais (4).

Nesse contexto, a legislação urbana no Brasil tem sido ineficaz, e a ocupação de áreas de fácil degradação ambiental tem sido comum nas cidades, sem falar na especulação imobiliária. Os planos diretores têm sido desrespeitados, agravando ainda mais os problemas ambientais urbanos. Algumas regiões do país têm demonstrado acelerado processo de industrialização aliado a uma forte expansão demográfica, trazendo, por um lado, o aumento da riqueza e do bem-estar social, e por outro lado, o agravamento dos problemas ambientais (5).

Logo, é importante conhecer o ambiente urbano assim transformado, para que se possa contribuir para a melhora e a manutenção da qualidade de vida (6). É necessário propor investigações que se contraponham às problemáticas desses impactos, desde a questão natural até as questões sociais e políticas (7). Dessa forma, o objetivo deste artigo é propor, a partir de uma reflexão sobre o conceito e as principais características do planejamento dos recursos hídricos, um índice de sustentabilidade da água urbana.

\section{MATERIAIS E MÉTODOS}

Neste trabalho destaca-se a importância do Rio Guandu no abastecimento urbano da região metropolitana do Rio de Janeiro. O Município do Rio de Janeiro está inserido na Região Sudeste do Brasil; sendo assim, os dados médios dessa região serão usados para fins de comparação com os do Município do Rio de Janeiro.

Originalmente, o Rio Guandu é uma continuação do Ribeirão das Lajes. A vazão natural do Guandu não passa de $20 \mathrm{~m}^{3} / \mathrm{s}$. Porém, na altura da barragem de Santa Cecília, localizada no Município de Barra do Piraí, a vazão do Guandu é aumentada para 300 $\mathrm{m}^{3} / \mathrm{s}$ por transposição das águas da bacia do Rio Paraíba do Sul. Próximo ao seu estuário, na Baía de Sepetiba, é chamado de canal de São Francisco.

O Guandu abastece 10 milhões de pessoas, o que justifica a necessidade de monitorar a qualidade de suas águas no que se refere à questão de saúde pública e de qualidade de vida.

A bacia do Rio Guandu enfrenta hoje a poluição provocada pelo lançamento de esgoto sanitário. O Rio Guandu recebe esgoto proveniente dos municípios do Rio de Janeiro, Nova Iguaçu, Japeri, Miguel Pereira e Seropédica, além do efluente da estação de tratamento de água (ETA) do complexo do Guandu, que lança uma grande quantidade de resíduos de origem metálica e inorgânica. Uma parcela pouco significativa desse esgoto sanitário recebe algum tratamento, tendo como destino final a Baía de Sepetiba. Com isso, observa-se a degradação dos ecossistemas aquáticos de toda a bacia hidrográfica, criando uma situação sanitária grave e provocando a degradação do rio, com o aumento, cada vez maior, da entrada de carga orgânica no sistema aquático.

A avaliação estratégica ambiental foi uma das primeiras ferramentas utilizadas para identificar, prever, avaliar e mitigar os efeitos biofísicos, sociais e outros relevantes para a sustentabilidade ambiental. Todavia, por razões diversas, tal procedimento ainda tem uso incipiente, sobretudo em países subdesenvolvidos. A figura 1 esboça diferentes parâmetros dos processos estratégicos de planejamento e avaliação ambiental (8), os quais subsidiaram a formulação, no presente estudo, do índice de sustentabilidade da água urbana.

Existem alguns modelos teóricos de sustentabilidade que podem auxiliar no desenvolvimento de um conceito de sustentabilidade urbana, fundamentalmente englobando um sistema sustentável dos recursos contidos no próprio território das cidades. Assim, a sustentabilidade das cidades abarcará, na sua gestão, procedimentos multissetoriais (9). Entretanto, abordamos neste artigo a sustentabilidade da água de consumo urbano pela ótica da crescente demanda por recursos hídri- 
FIGURA 1. Parâmetros de elaboração estratégica no planejamento ambiental

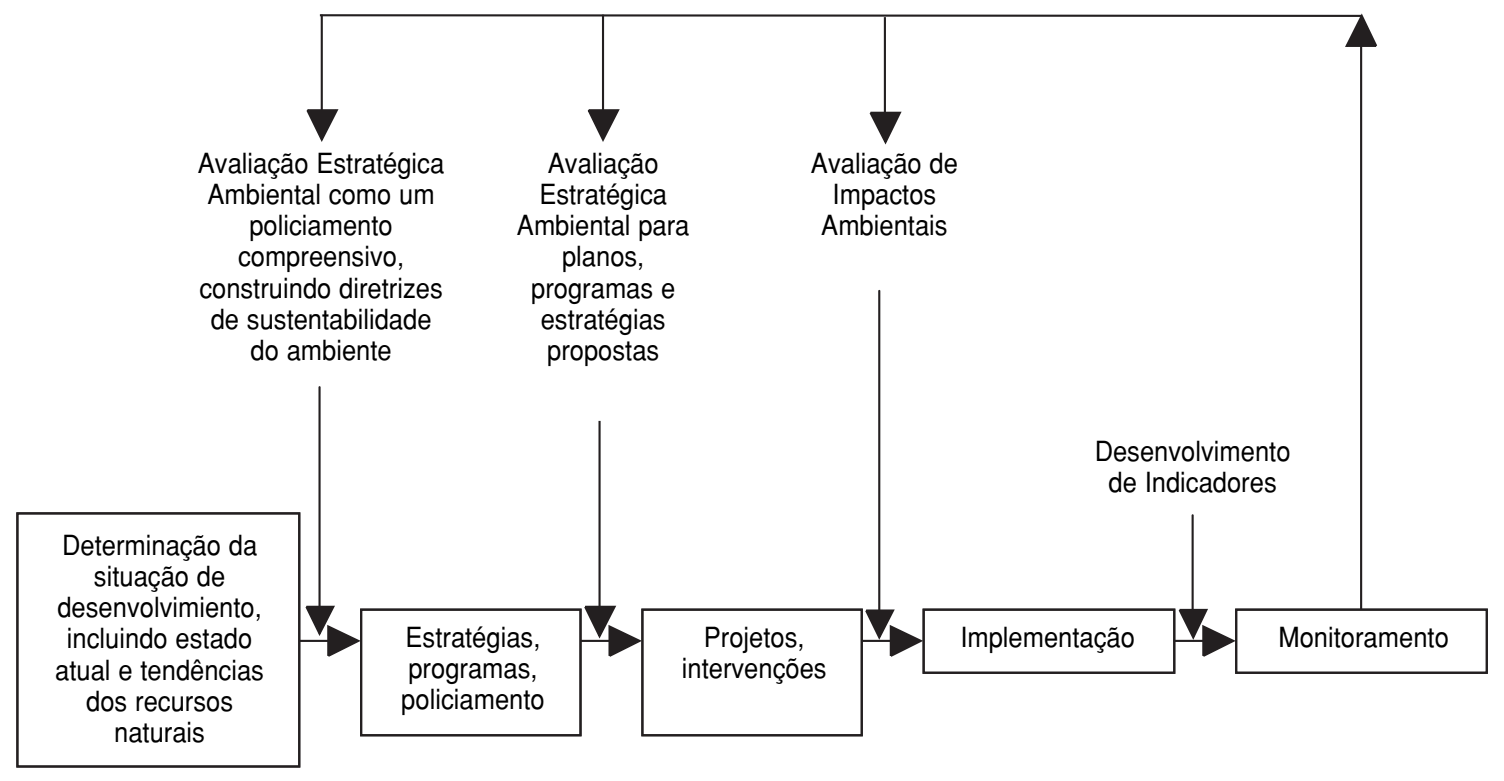

cos, estabelecida através de um índice, fazendo com que, a partir da constatação de sua escassez, a água passe a ser considerada como um recurso natural com valor econômico, estratégico e social (10).

\section{Estabelecimento do índice de sustentabilidade da água urbana}

A sustentabilidade ambiental dos sistemas de água urbana depende de mecanismos multidisciplinares e integrados em tempos e escalas variadas. Entre as características necessárias aos indicadores urbanos (11) destacam-se: ser de fácil entendimento pelos tomadores de decisão; incorporar o interesse de um ou mais grupos de tomadores de decisão; ser mensurável, com dados disponíveis para uso imediato dentro da própria cidade ou em nível nacional; ser claramente relacionados aos objetivos das políticas urbanas e passíveis de mudança através do uso dos instrumentos políticos; e integrar, sempre que possível, os temas econômicos, sociais e ambientais relevantes.

Para o estabelecimento do índice de sustentabilidade da água urbana, incorporou-se o conhecimento sobre os efeitos provocados pelo uso inde- vido da água urbana, a quantidade e a qualidade dessa água, sua influência sobre a saúde ambiental da população e o atendimento da população pelo serviço de esgotamento sanitário. Estabeleceu-se que o índice deveria refletir a correta manutenção de ecossistemas aquáticos e a proteção da saúde humana, podendo servir como um instrumento de controle ou simplesmente como um indicador de sustentabilidade da água urbana.

Na definição de um índice de sustentabilidade da água urbana que proteja os ecossistemas aquáticos e a saúde ambiental, devem-se utilizar indicadores que traduzam os usos múltiplos dos recursos hídricos e todos os conflitos decorrentes desses usos. Uma proposta de determinação de um índice de sustentabilidade pode ser feita a partir dos índices de qualidade da água (IQA) existentes (12). Esses índices são bastante úteis para verificar a qualidade da água em corpos d'água, permitindo, ainda, comparações entre diferentes corpos d'água. $\mathrm{O}$ índice de qualidade varia de 0 a 100 , sendo que, quanto maior for o valor do IQA, melhor será a qualidade da água. No procedimento para o estabelecimento do IQA, deve-se considerar qual o uso que se deseja para o corpo d'água, de- terminar quais parâmetros melhor descrevem o uso definido e estabelecer relações entre os parâmetros e a qualidade da água, pontuando, dentro de uma escala, os parâmetros mais importantes e estabelecendo um sistema de pesos para que, através de uma média ponderada, consiga-se uma nota para a qualidade da água do curso d'água em questão.

Assim, neste trabalho, optou-se por desenvolver um índice de forma a subsidiar uma primeira visualização do contexto ambiental urbano, usando dados consolidados de indicadores. Com esta metodologia, criou-se um índice considerando os parâmetros que descrevem a sustentabilidade da água urbana. Selecionaram-se, criteriosamente, 11 indicadores, com base nos parâmetros dos serviços de água e esgoto do Sistema Nacional de Informação sobre Saneamento (SNIS) (13) para os anos-base 2000 e 2001. Os indicadores considerados são listados na tabela 1. O índice de sustentabilidade da água urbana (ISAU) pode ser calculado como: ISAU = produto $\left(\mathrm{I}_{i}^{p i}\right)$, onde $I_{i}$ é a nota para cada indicador $i$, variando de 0 a 100; e $p_{i}$ é o peso para cada indicador $\mathrm{i}$, sendo $\sum_{i=1}^{n} p_{i}=1$, onde $\mathrm{n}$ é o número de indicadores considerados. 
TABELA 1. Indicadores usados para o cálculo do índice de sustentabilidade da água urbana, Município do Rio de Janeiro (RJ) e Região Sudeste, Brasila

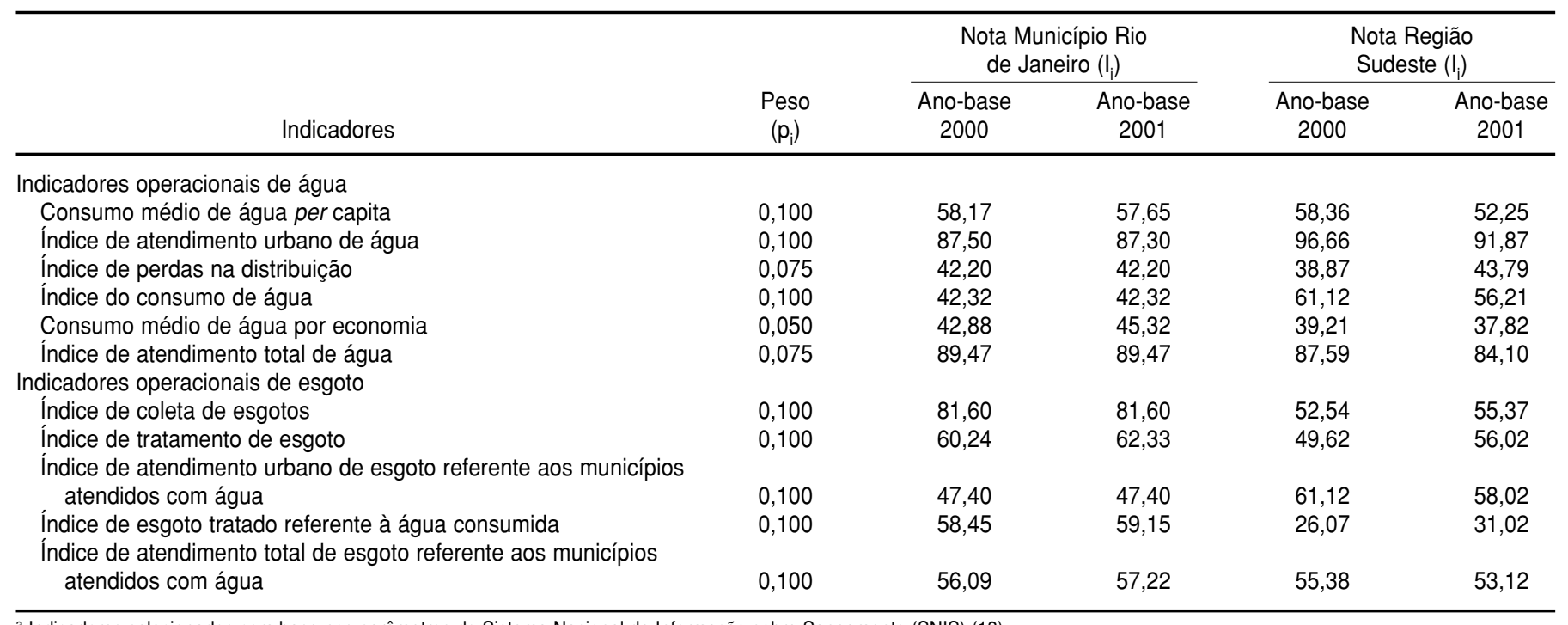

${ }^{a}$ Indicadores selecionados com base nos parâmetros do Sistema Nacional de Informação sobre Saneamento (SNIS) (13).

Os pesos de cada indicador refletem a sua importância para o ecossistema urbano. Neste trabalho, os valores relativos aos pesos foram arbitrados, considerando metade para os indicadores operacionais de água e a outra metade para indicadores operacionais de esgoto. O SNIS possui 20 indicadores operacionais de água e sete de esgoto; o ISAU reflete essa distribuição, considerando que foram escolhidos seis indicadores para a água e cinco para o esgoto. Os indicadores que se relacionam diretamente com a qualidade da água urbana tiveram um peso maior em relação àqueles cuja relação não é direta. No entanto, deve-se procurar estabelecer uma discussão, junto à comunidade científica, quanto à definição dos pesos, e as pesquisas nesse sentido devem ser incentivadas.

As notas usadas para cada indicador foram obtidas diretamente quando o indicador era dado em porcentagem (variação entre 0 e 100). Para os indicadores de consumo médio per capita de água e de consumo médio por economia (quociente entre volume de água consumido menos volume de água tratada exportada e a quantidade de economias ativas de água) (13), cuja unidade não é percentual, foram criadas relações capazes de descrever tais
FIGURA 2. Variação da nota atribuída ao indicador consumo médio per capita de água utilizado no cálculo do índice de sustentabilidade da água urbana

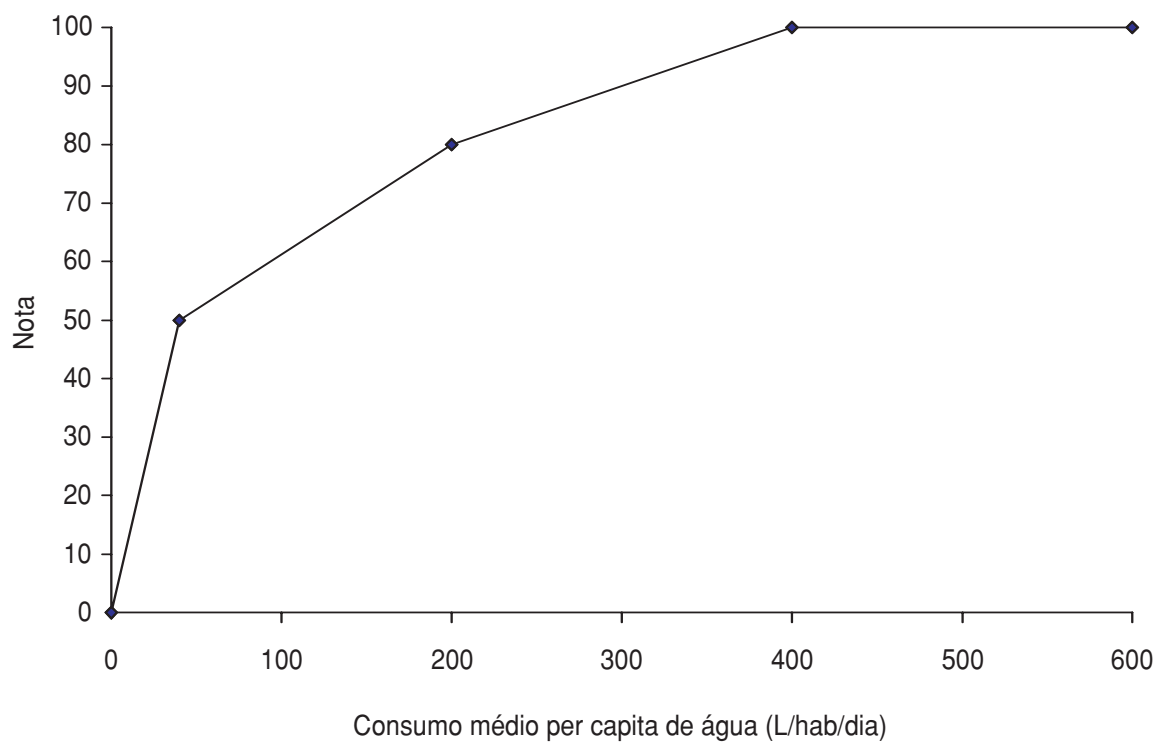

variações. Para o caso do consumo médio per capita, a nota máxima (100) correspondeu ao consumo de 400 $\mathrm{L} / \mathrm{hab} /$ dia ou mais. Para um consumo de $40 \mathrm{~L} / \mathrm{hab} /$ dia, a nota correspondente é 50, considerando que este é o valor definido pela Organização Mundial da Saúde (OMS) como sendo o mí- nimo necessário para que uma pessoa possa viver em condições razoáveis. A variação da nota deste indicador pode ser observada na figura 2. No caso do consumo médio por economia, foi estabelecida uma relação linear, considerando-se, como valor máximo, o volume disponibilizado por economia. 


\section{RESULTADOS}

A tabela 1 mostra os valores consolidados de pesos e as notas para cada indicador usado nas avaliações do índice de sustentabilidade da água urbana. Foram definidos, então, critérios de qualidade a partir de valores do ISAU: um valor entre 0 a 19 indica qualidade péssima da água; de 20 a 36, qualidade ruim; de 37 a 51, qualidade aceitável; de 52 a 79, qualidade boa; e de 80 a 100, qualidade ótima.

Os índices de sustentabilidade da água urbana do Município do Rio de Janeiro, considerando os anos-base 2000 e 2001, foram de 58,99 e 59,57 (qualidade boa), respectivamente. Pode-se notar que houve uma elevação do índice do ano de 2000 para 2001, ou seja, um ligeiro aumento na qualidade do serviço.

Considerando que o índice de sustentabilidade da água urbana proposto permite comparações entre diferentes regiões, foi feito o cálculo para a Região Sudeste, a partir da média regional dos 11 indicadores envolvidos, considerando todos os municípios da região. Os valores para o Sudeste foram de 54,26 e 54,53 (qualidade boa) para os anos-base 2000 e 2001, respectivamente. Nota-se, assim, que o $\mathrm{Mu}-$ nicípio do Rio de Janeiro teve uma qualidade de serviço melhor do que a média regional. Percebe-se, ainda, que a melhora no serviço foi maior no $\mathrm{Mu}-$ nicípio do Rio de Janeiro do que na Região Sudeste.

\section{DISCUSSÃO}

O uso de indicadores na avaliação de impactos ambientais é um instrumento de política ambiental, formado por um conjunto de procedimentos capaz de assegurar, desde o início do processo, um exame sistemático dos impactos ambientais de uma ação proposta e de suas alternativas (14). Esta definição evidencia que a avaliação de impactos ambientais subsidia o processo de tomada de decisão e se atém à ação proposta-políticas, planos, programas e novas tecnologias. $\mathrm{O}$ ajuste estrutural da sustentabilidade da água e do ambiente exige critérios metodológicos analíticos que embasem e fundamentem o uso de indicadores ambientais (15).

Entretanto, quanto às questões urbanas da água, a poluição é um dos aspectos mais preocupantes. Além das perdas, do desperdício e da falta de investimento nas redes de distribuição de água potável, a poluição tem gerado situações críticas quando a conjuntura natural é desfavorável. Os maiores responsáveis são os esgotos domésticos, os efluentes industriais não tratados e o uso intensivo de insumos químicos na produção agrícola.

Na realidade, apenas 10\% dos países sofrem de escassez quantitativa de água. Nos demais, dos quais o Brasil faz parte, o maior problema é a qualidade da água. A situação fica, muitas vezes, insustentável, na medida em que se retira mais água ou se polui mais rápido do que a capacidade natural de recuperação dos mananciais. $\mathrm{Ou}$ seja, a natureza não acompanha a velocidade com a qual as ações antrópicas agridem o ambiente natural; assim, o volume de água potável por habitante torna-se cada vez menor.

$\mathrm{Na}$ seqüência da Conferência das Nações Unidas sobre Meio Ambiente e Desenvolvimento, a ECO-92, realizada no Rio de Janeiro, em 1992, tornou-se muito forte a exigência do conceito de sustentabilidade nas políticas de gestão ambiental. Com esse conceito, a escala de longo prazo regressou às metodologias de decisão. A expressão visível dessa evolução é a adoção de um conceito de sustentabilidade ambiental integrador e estratégico-estratégia de que é paradigma a Agenda 21, com um permanente processo de monitorizarão, definição de objetivos, formulação e implementação de medidas.

A gestão ambiental é inerente à conduta humana quando sujeita ao envolvimento ambiental. As escalas espaciais e temporais das intervenções são variáveis, indo desde um padrão mínimo individual, passando pelos grandes projetos coletivos até a escala continental, marcando, para os séculos vindouros, a diferença e as alterações provocadas. Nas políticas públicas de recursos hídricos, o planejamento ficou muito associado ao desenvolvimento, onde o pragmatismo recomenda que o planejamento seja valorizado pela possibilidade de se atingir mais rapidamente, e com benefícios evidentes, nomeadamente econômicos, novos patamares definidos como objetivos.

A crescente demanda por recursos hídricos, tanto em quantidade como em qualidade, aumenta a disputa pela sua utilização. A percepção da escassez faz com que a água passe a ser considerada um recurso natural com valor econômico, estratégico e social. Apesar do processo de modificação do ambiente não ocorrer de forma linear, a escassez tem significativa influência na velocidade de mudança do ambiente e no seu grau de complexidade (10). Por isso, devemos ter como meta futura a elaboração de modelos que possam fundamentar objetivamente as observações a que nos propomos. Embora os sistemas de purificação engendrados pelo gênio humano não contenham a dimensão ecológica e a sustentabilidade do ciclo hidrológico, eles permitiram o desenvolvimento de uma nova fonte de recursos hídricos, atribuindo uma dimensão ecoeficiente à água.

Até a década de 1990, cerca de $85 \%$ do esgoto produzido pelo Município do Rio de Janeiro não recebia tratamento adequado (16). Desde então, um programa de recuperação ambiental da região, que prevê a construção e a ampliação de estações de tratamento de esgoto, de emissários e redes coletoras, além de investimentos nas áreas de macrodrenagem e de resíduos sólidos, beneficiando áreas de alta concentração urbana, inclusive favelas, vem gerando resultados. A evolução temporal do índice de sustentabilidade da água urbana mostra que o aumento no atendimento total e a maior cobertura do tratamento de esgoto trouxeram melhorias. Considerando que o Município do Rio de Janeiro vem controlando principalmente as fontes pontuais de poluição e melhorando a eficiência das plantas de tratamento de esgoto doméstico, é de se esperar um aumento no índice. No entanto, muito precisa ser feito no sen- 
tido de garantir uma melhor qualidade da água urbana, e conseqüentemente, um aumento no valor do índice de sustentabilidade da água urbana.

Tendo-se em vista que a avaliação estratégica ambiental dos sistemas urbanos de água é essencialmente uma análise dos recursos naturais e de seus valores, dinâmicas, pressões e fatores relevantes, seguida por uma avaliação das ligações desses fatores com os índices de sustentabilidade, o sucesso dos planos de uso eficiente e reuso da água urbana depende da maneira e da profundidade com que algumas ações são implementadas, podendo-se destacar os critérios adotados para avaliar as alternativas de uso racional e eficiente da água; a definição adequada de estratégias de coleta, lançamento e tratamento dos esgotos; e a importância atribuída à escassez hídrica e ao equilíbrio dos ecossistemas.

\section{CONCLUSÕES}

A consideração operacional da sustentabilidade está ainda mal definida. É um conceito simples de explicar, mas muito difícil de aplicar. No domínio ambiental, em particular em termos dos recursos hídricos, várias tendências ensaiam a apropriação do conceito de forma a incorporá-lo a metodologias já existentes. É o caso da economia, através da internalização de custos, tentando considerar os valores ambientais como sendo semelhantes a outros valores de mercado (17). Assim, o método aqui proposto tem por objetivo influenciar indiretamente a decisão dos gestores, consumidores, utilizadores da água e investidores.

São de grande relevância os indicadores ambientais na análise da qualidade ambiental urbana. Segundo estudos anteriores (18), os indicadores ambientais são modelos que descrevem as fórmulas de relação das atividades humanas com o ambiente como fonte de recursos, depósito de rejeitos, suporte da vida humana e fonte de biodiversidade. Tais indicadores podem se referir ao estado físico ou biológico do mundo natural (indicadores de estado), às pressões das atividades humanas que causam modificações nesses estados (indicadores de pressão) e às medidas políticas adotadas como resposta a tais pressões, na busca da melhora do meio ambiente ou da mitigação da degradação associada às questões urbanas. Deve-se ressaltar que os indicadores ambientais auxiliam na análise da qualidade ambiental urbana, que pode ser boa ou ruim, dependendo de como a pressão humana se impõe no meio ambiente.

Várias interpretações estão consolidadas no dia-a-dia, podendo-se destacar a visão de que o acesso à água deveria se dar de forma gratuita ou por preço irrisório (considerando que a água está disponível no ambiente), embutindo-se nessa concepção, ainda, o descaso com as águas servidas, que contaminam os corpos receptores; e, por outro lado, a necessidade premente de investimentos no serviço de fornecimento, sobretudo em linhas novas de uso e reuso de água para fins menos nobres (setores industriais, etc). Mais do que planos para a concretização de medidas objetivas, a gestão da água urbana deve ser um instrumento de mitigação antecipada de efeitos negativos previstos, servindo para conter os riscos relativos à água.

A sociologia, por seu turno, defende que a tomada de decisões ambientais reflete preocupações e compromissos sociais. A teoria social está crescentemente interessada nos problemas que o ambiente colocará, no futuro, aos cidadãos, mais do que nos problemas que estes criarão ao ambiente. Um aspecto importante na atual prática de planejamento é o papel das sociedades organizadas na percepção, consciência e interpretação da realidade. Os eventuais efeitos de uma sociedade de racionalidade incompleta e da percepção e construção social dos problemas ambientais têm particular importância na faceta relativa à participação pública na elaboração, aceitação e gestão dos planos. Através dos planejamentos ambientais, incluindo o planejamento dos recursos hídricos, a sociedade poderá definir trajetórias de desenvolvimento em função dos fatores econômicos, sociais e culturais.

\section{REFERÊNCIAS}

1. Rebouças AC. Água doce no mundo e no Brasil. Em: Rebouças AC, Braga B, Tundisi JG, orgs. Águas doces no Brasil: capital ecológico, uso e conservação. São Paulo: Editora Escrituras; 1999. Pp. 1-36.

2. Levine D. Atlas novo milênio de população mundial. Disponível em: http://www. novomilenio.inf.br/porto/mapas/nmpop. $\mathrm{htm}$. Acessado em abril de 2005.

3. Tucci CEM, Hespanhol I, Cordeiro Netto OM. A gestão da água no Brasil: uma primeira avaliação da situação atual e das perspectivas para 2025 [relatório]. Global Water Partnership: 2000. Acessível em: http://www.unb. $\mathrm{br} / \mathrm{ft} / \mathrm{enc} /$ recursoshidricos/relatorio.pdf. Acessado em abril de 2005.
4. Pesce SF, Wunderlin DA. Use of water quality indices to verify the impact of Cordoba City (Argentina) on Suquia River. Water Res. 2000; 34(11):2915-26.

5. Schenini PC. Avaliação dos padrões de competitividade à luz do desenvolvimento sustentável: o caso da Indústria Trombini Papel e Embalagens S.A. em Santa Catarina. Florianópolis: UFSC; 1999.

6. Silva MLG. Análise da qualidade ambiental urbana da bacia hidrográfica da Lagoa da Conceição. Florianópolis: UFSC; 2002.

7. Coelho MCN. Impactos ambientais em áreas urbanas-teorias, conceitos e métodos de pesquisa. Em: Guerra AJT, Cunha SB, orgs. Impactos ambientais urbanos no Brasil. Rio de Janeiro: Bertrand Brasil; 2001. Vol 1. Pp. 19-45.

8. Kessler JJ, Van Dorp M. Structural adjustment and the environment: the need for an analytical methodology. Ecol Econ. 1998;27(3): 267-81.

9. Zancheti SM. O desenvolvimento sustentável urbano. Disponível em: http://www.urban conservation.org/textos/desenv_sustentavel. $\mathrm{htm}$. Acessado em abril de 2005.

10. Scare RF. A escassez de água e mudança institucional: análise da regulação dos recursos hídricos no Brasil. São Paulo: USP; 2003.

11. Veríssimo M, Rosa JW, Ribeiro MCL, Giacomoni MH, Pinha PRS. Indicadores urbanos para sustentabilidade dos sistemas hídricos. 
Estudo de caso: Distrito Federal. Universa. 2001;9(2):245-78.

12. Porto MFA. Hidrologia ambiental. São Paulo: ABRH/EDUSP; 1991. (Coleção ABRH).

13. Brasil, Secretaria Especial de Desenvolvimento Urbano da Presidência da República. Sistema Nacional de Informação sobre Saneamento (SNIS). Disponível em: http://www. snis.gov.br/ diag_2000.htm e http://www. snis.gov.br/ diag_2001.htm. Acessado em junho de 2002.

14. Soares BES, Ferreira AP. Desenvolvimento sustentável e biodiversidade: gestão reacional e ecológica dos recursos ambientais. Biotecnologia Ciencia Desenvolv. 2004;33:72-5.

15. Lundin M, Morrison GM. A life cycle assessment based procedure for development of environmental sustainability indicators for urban water systems. Urban Water. 2002;4(2): $145-52$.

16. Costa H. Uma avaliação da qualidade das águas costeiras do Estado do Rio de Janeiro. Rio de Janeiro: FEMAR; 1998.

17. Mierzwa JC, Hespanhol I. Programa para o gerenciamento de águas e efluentes nas in- dústrias, visando ao uso racional e à reutilização. Eng Sanit Ambient. 2000;4(1):11-15.

18. Herculano S. A qualidade de vida e seus indicadores. Ambient Soc. 1998;1(2):77-100.

Manuscrito recebido em 11 de novembro de 2003. Aceito em versão revisada em 25 de fevereiro de 2005.

ABSTRACT Objective. To propose an urban water sustainability index based on indicators that may serve as a foundation for developing planned actions concerning water resources. Methods. Eleven indicators (covering aspects of water and sewage) were selected based on the parameters of Brazil's National Water and Sanitation Information System (Sistema Nacional de Informação sobre Saneamento) for the years 2000 and 2001. A score and a weight were assigned to each indicator. Based on that, the urban water sustainability index (UWSI) was calculated, using the formula UWSI = product $\left(I_{i}^{p i}\right)$, where $I_{i}$ is the score attributed to each indicator $i$, ranging from 0 to 100 , and $p_{i}$ is the weight for each indicator $\sum_{i=1}^{n} p_{i}=1$, where $n$ is the number of indicators considered). The lowest water quality is indicated by an index value of 0 , and the best by an index value of 100 .

Results. The urban water sustainability index for the city of Rio de Janeiro was 58.99 in 2000, and it rose to 59.57 in 2001, indicating water of good quality in both those years.

Conclusions. The improvement in the quality of the water resources between 2000 and 2001 in the city of Rio de Janeiro is possibly the result of the implementation, in the 1990s, of an environmental management program in the Rio de Janeiro region. Environmental planning that includes rational use of water resources and methods to prevent their destruction is crucial to sustaining society.

Keywords Water supply, water pollution, water purification, environmental health, waste management, conservation of natural resources. 